Julie Huang Xinet 2560 Ninth Street, Suite 312,

Berkeley CA 94710, USA Tel: +18450555

E-mail: julie.huang@xinet. com

\title{
Papers
}

\section{Assessing the value of DAM systems for advertising agencies}

\section{Ron Roszkiewicz}

is an independent consultant and software developer based in the San Diego area. Ron's 20-year career includes a variety of development positions at Dainippon SCREEN, Scitex, DK\&A, and Pound Hill Software.

\section{Keywords: DAM, advertising agencies, ROI, best practices}

Abstract Advertising agencies make money providing innovative solutions to promote their clients' brands and services through an increasingly wide spectrum of media. Innovative creative solutions today typically involve innovative technology to support its creation. But in contrast to the readiness to adopt new technology that assists in creating artwork or automates formatting of final output for production, the adoption of systems to manage the creative process has not been as rapid. In the final analysis, it is all about time to market and the pressure from clients to accelerate the delivery schedule is ever-present. The challenge is to blend a creative workflow with the digital production workflow to achieve a satisfactory $\mathrm{ROI}$ - while minimizing disruption and keeping up with a manageable maintenance regimen - that fits the objectives of the organization. Journal of Digital Asset Management (2007) 3, 116-123. doi:10.1057/palgrave.dam.3650077

\section{INTRODUCTION}

This paper provides a basis for justifying the adoption of a DAM system. There are a number of ways to calculate ROI, each of which takes into account some aspect of:

- Pre-purchase planning;

- System installation and integration;

- Post-installation system adoption.

Adopting a DAM system is as much about changing the culture of a company as it is about changing the technology. The considerations, calculations and best practices used by successful DAM system sites are described below.

\section{TECHNOLOGY IN CONTEXT}

The past ten years have been particularly interesting for the advertising industry. The emergence of the web provided new revenue opportunities as well as the potential for workflow enhancement. Taking a high-level view of all that has come about as a result of the development of a commercially available Web, one can characterize the past decade as a period of transactional productivity gains.
Every file downloaded, form filled out, item purchased and message sent is a transaction: relatively easy stuff to automate and easy to quantify in terms of cost savings and increased productivity. In the beginning, data repositories supported these transactions as a component in hybrid digital workflows. The net result of the ongoing development of these transactional productivity solutions is a slew of new technologies (including web services, XML metadata, open standards such as RDF, DHTML and PHP) that support many variations of commercial transactions.

A by-product of these transactional productivity developments is the establishment of an infrastructure to support the current wave of advertising agency workflow. This wave is characterized by interactive productivity solutions that may include transactions but more importantly provide new ways for agencies and clients to interact with each other in the creation, review and approval processes.

The reality is that design will continue to be subjective and regardless of how sophisticated technology becomes it will only support and validate that which humans tell it to expect. 
Instead of a totally automated design workflow, the ideal vision is one that is automated where appropriate and supports the interaction and management of interactivity regardless of scale or gateway. The ideal system is operating system and device neutral.

\section{ROI IN CONTEXT}

Quantifying the ROI of a DAM system in the advertising agency environment is useful even if in the end it is only a small part of the total story. Consider the competitive context in which these systems exist:

Cost of doing business (CDB): Calculate, if only anecdotally, the cost of not adopting a DAM system, improving workflow or taking advantage of a new revenue opportunity the technology enables.

Total cost of ownership (TCO): Determine the associated cost of managing and maintaining a system within a creative workgroup or through an IT department.

Total cost of adoption (TCA): A broader point of view requires examining the cultural implications of changing the way a company does business. When a post-mortem is carried out after a new system is installed, the factors that lead to a successful or failed installation are often the ones that should have been included in a TCA calculation. Avoiding failure in introducing a system is normally a result of positive TCA considerations and procedures.

ROI can also be broken down into two distinct and related perspectives:

Tactical ROI relies on measured savings from increased productivity in the department(s) where a system will be used.

Strategic ROI relates to the positive or negative impact on the broader activities of the company.

No two companies are exactly alike. Even two departments or subsidiaries in the same corporation will often have vastly different workflows. Introducing a system of any type into these environments is often the beginning of formalizing, or even standardizing, a workflow.

Expertise in "how the business works" rests with one or at most with a few persons. Replacing this human intelligence with machine intelligence cannot instantly result in productivity gains. In the long run, however, codifying the storage and management of valuable intellectual property overshadows the relative stability of the status quo. Especially if the company's success means a flood of new assets to manage and a more complex workflow. What might have worked in the smaller company is quickly eclipsed as the system scales up.

The following discussion provides some considerations about tactical and strategic ROI, CDB, TCO, TCA and some other best practices to be aware of in making a case for adopting a system. It is almost universally accepted today that DAM systems provide short-term ROI and long-term workflow advantages. The primary reasons for failure to see a positive return are poor planning, lack of understanding of business objectives and poor change-over management when moving from an existing workflow to the new system.

\section{COST OF DOING BUSINESS}

When all is said and done, the control over digital assets is becoming more than a useful workflow enhancement, it is becoming a corporate imperative. First of all there are compliance issues. Sarbanes-Oxley is the accounting equivalent of $\mathrm{Y} 2 \mathrm{~K}$. The repercussions on how we value intellectual property and how we account for it as a managed resource are felt throughout the organization and eventually throughout the industry. In times past, when intellectual property was managed either through a database of records or in physical form, this was less of an issue. Today, CEOs must take responsibility for accurately disclosing this often-substantial asset. Of course, this cannot be done unless there is a system in place to authenticate the existence and content of digital assets. DAM systems provide this controlled environment.

Another area of importance is feeding a web presence. All advertising agencies must maintain a digital portfolio, a place for clients to upload and download artwork, and a way to support web conferencing for interactive project review. In the best of circumstances the system that provides this level of functionality is integrated into the data repository.

The reach of the internet also facilitates global multi-channel marketing like never 
before. Managing a combination of localized custom websites and providing access to localized marketing collateral and original art along with permission set associated with each client are nearly impossible without the support of a robust DAM system.

\section{UNDERSTANDING THE TCA}

A successful DAM system installation, in theory, is measured by a positive ROI. Unfortunately, ROI calculations often prove useless because there are no calculations for the social engineering that is part of the workflow overhaul. Even with the best intentions, the expectations of the vendor, management and users are often unreconciled before a purchase agreement for a new DAM system is signed.

There is no one formula that can adequately predict or model the success or failure of an installation, but it is easy to build a successful model of an installation and have it fail for reasons that might not be factored into a typical ROI, or TCO calculation. For this reason, TCA is this writer's preferred higher level view of all of the social and technological input that must be taken into account and then managed in order for a true ROI to be realized.

Understanding what the TCA factors are and how to manage them is best researched through best practices. As more and more systems are installed and case histories compiled, the best practices' knowledge-base increases. It is possible to foresee the effects that implementing a new system will have on a workgroup - or on the enterprise - and subvert potential resistance.

Some of the responsibility for success and a positive ROI/TCA lies with the vendor and some lies with the customer. The important point here is that if resistance to adoption is inevitable, formal procedures must be put in place to resolve it. Communication is key but only through a formal procedure. Another key ingredient is the environment the system creates. An intuitive, high-performance and easily integrated environment that fits easily into an existing workflow is one that will be embraced and not seen as a threat to anyone in the creative or production hierarchy. A system that grows organically and is flexible enough to adapt to new requirements that can transcend just being a useful workflow solution to become a tool for modeling and building new revenue streams.

\section{Planning Checklist}

$\checkmark$ Workflow discovery

$\checkmark$ Research products and solutions

$\checkmark$ Consider collaboration features

$\checkmark$ Evaluate change-management requirements

\section{PLANNING FOR A DAM SYSTEM}

Good planning and execution leads to successful implementation and positive ROI. It begins with crafting a business plan to justify the system and get executive buy-in and ends with a postmortem to wrap up the project. Effective communication of objectives and status are critical at every stage of project planning and execution. A thorough understanding of the existing workflow, open and regular paths for authorized information to flow and realistic milestones typically result in reduced risk. Expect change and learn to manage it. Expect resistance. Fear of the unknown can only be reduced over time by competent project management and communications. Putting in place procedures for communicating and decision-making is also a great way to reduce stress personally and organizationally (Figure 1).

The following topics explain the best practices for dealing with the different stages of project planning and execution typically found in a DAM system installation.

\section{WORKFLOW DISCOVERY}

The most important part of the project will be planning and initial discovery. This is the time when a full evaluation of the existing workflow takes place; key stakeholders are identified and assigned to the project team; a business plan and system specification is drafted; and initial stabs are taken at gathering existing metadata, important interoperability requirements and IT issues. All of this, plus getting senior management buy-in for the project, are essential to the success of DAM system implementation.

If planning how the system will be integrated into the existing workflow is not dealt with at this point but is left for later, it will be a disaster. At that point, power shifts to the users who may 
TCA FACTOR S

To calculate the TCA of a DAM system in an advertising agency environment, quantify the man hours involved in:

- Reviewing and evaluating the current workflow

- Identifying points of personal contact and "flow" between ad agency department, agency vendors and clients

- Ingesting metadata and digital asset files into new system

- Running parallel workflows during installation and testing of system

- Training system administration

- Training agency personnel in all touch-point departments

- Training clients

- Ongoing training as feature sets are upgraded and as new users are added to agency, vendor, and client sites

Figure 1: TCA factors

accept or reject features that do not match up to a long-practiced agency-standard workflow. Many systems wind up in dusty utility closets for just that reason.

Workflow discovery must include interviews with staff to determine tasks and responsibilities; flow charts of existing workflows and touch points to other departments; and a gathering of existing metadata in the form of project names, descriptions and stakeholders who touch each project.

\section{SEEK OUT THE MOST COMPLETE SOLUTION}

In the perfect world the perfect system would be available off-the-shelf, require minimum setup and come at a cost that would not break the budget. Unfortunately, modern systems require a complex architecture to support a robust database foundation as well as many interoperability requirements. The key benchmark for end-to-end functionality is in evaluating the total content lifecycle. A DAM system must provide functionality for the following content lifecycle stages: creation, management, distribution and storage.

Make sure, too, that open standards are part of the solution. For example, if a system relies on XMP-formatted metadata packets, it will be compliant with a standard that is emerging in the content-creation community. In the life of any system, there will come a time when interoperability is critical to further automation of the system. This may be the result of the need to link to other workgroups or to other rights management or production servers. Both XMP and SQL are established standards that must be supported in systems available today. When considering competing systems, overlay the stages of content lifecycle over each system and identify how each stage will be supported by some functionality in each system.

Content lifecycle - Creation: Be sure that desktop applications are supported and the access to the DAM system is optimized by easy-to-use XTensions and plug-ins that help find and place assets with a minimum of clicks. The first place asset repositories feed is the creative process. Any resistance to using the system on the part of creative users breaks down the control and integrity of a DAM-based workflow.

Content lifecycle - Management: How assets are stored, processed and presented to the user are important functions to be understood during the product review process, as is the ascertainment that a rich metadata environment is in place to make searches efficient. A comprehensive DAM system should be compiling a history over the life of the asset during its residence in the system and long after it is archived.

Content lifecycle - Distribution: A system's flexibility to deliver access to the many channels of asset creators and users is critical. How easy is it to provide or edit permissions for a user? 
Does the system handle repurposing automatically based on users' requests? How easy is it to set up access for a new client? Vertical integration means not only that is the functionality available but also that it can be used by the intended audience. It is very common today for the IT department in large and small companies to push back the responsibility of managing digital assets to the department that creates them. Administering the system should be possible without hiring additional technical staff.

Content lifecycle - Storage and Archiving: Every system either provides a built-in archiving system and/or has extensions to provide interoperability with common archiving systems. Near-line or offline storage is an important stage of content lifecycle management. Considering the sheer volume of digital assets retained by some agencies for repurposing, revision and localization, it is expected that assets will be stored offline. The process for retrieving them must be integrated into the core system. Once again this is necessary to maintain the control of asset movement and the integrity of the asset itself.

Does the System Promote Collaboration? Ad agency work is collaborative and while applications that support true collaboration are still a bit of a dream, the system you buy should be putting in place functions or standards that will promote collaboration. Currently, you can expect a DAM system to be a hub for content approval and proofing.

Review for content: Most of the time reviews are carried out for content. Body copy, prices, SKUs must be checked and rechecked to inspect whether the file is analog or digital. It is important to have the tools to review these files both in their native format as well as in the PDF format. The ideal system should allow reviews to take place without a need for the originating application. This means that clients, marketers, account executives and sales representatives would be able to review work wherever they are and without having the same application, or worse, the same application configuration.

Proof for quality: The most efficient way to proof art is on a screen. Without this functionality true remote collaboration will not be possible. New ways to synchronize color spaces on different but linked screens are emerging to solve the soft-proofing dilemma that has persisted for many years. As new schemes emerge they should build on existing core layout review functionality and manage the review process from within the workflow, and not force users to go outside of it and once again break down the control a DAM system provides.

Change management: Every proposed installation involves moving from one system to another. This can mean moving from an analog to a digital one or from digital to digital. It can involve moving people around or even out the door. There will be implications for training requirements. There will also be a need for postinstallation support and system monitoring.

One of the easiest ways to alienate staff is to leave them out of the loop regarding the plan and objectives of the new system. It is critical to overcome resistance to a new way of doing things with the key stakeholders and to use this as leverage to communicate with and win over the remaining user base. An alienated pool of creative and production people will guarantee failure.

Change management is an organic process and is every bit as important as risk management in the life of a project. It should be a formal process that takes what is learned during the initial workflow discovery stage, continues through installation and is synthesized into communications that are part of user testing of the system and user training (Figure 2).

\section{TACTICAL ROI}

Productivity typically equates to reducing keystrokes with the eventual result in a reduced head count or increased capacity. This truism is first of all the easiest case to make in building a business plan for adopting a new system. In lieu of literally counting keystrokes, one can draw upon some studies carried out by industry analysts and consultants. Numbers alone do not tell the whole story, especially in a highly charged environment such as an advertising agency. In such a setting it is important to explore the consequences of working with and without a controlled data environment. 


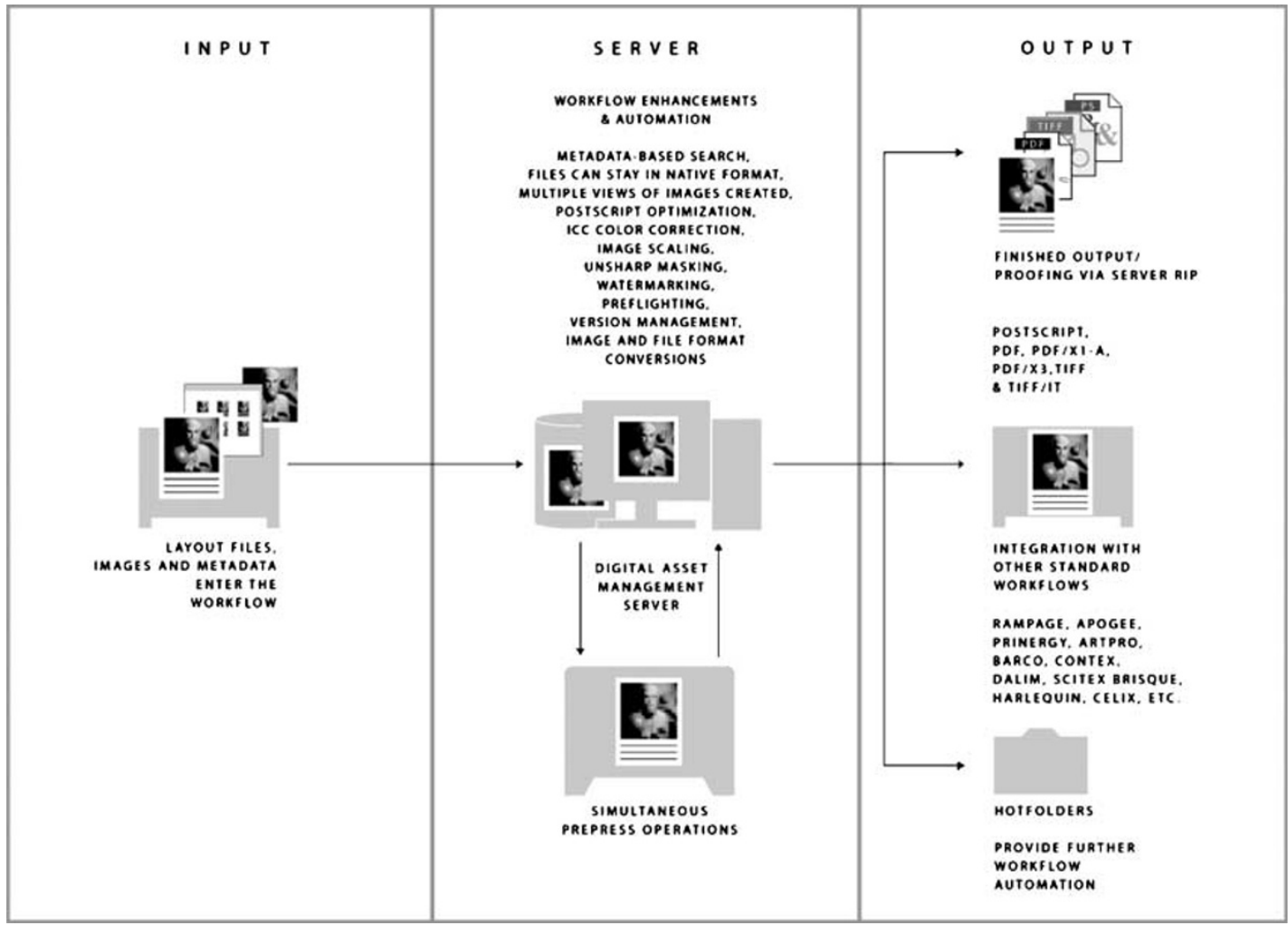

Figure 2: Content lifecycle and production

\section{TIME LOST ACCESSING DATA}

The most commonly used estimate of the amount of time used to search for a file is three minutes and the searched-for file is not found 40 per cent of the time. The estimate of the number of searches done in a typical year is 2,500 per person. That equates to 15.5 man-days in 200 for searching. Obviously, searches will continue regardless of whether or not a system is in place and time doing it will continue to be spent.

A typical consequence of not having a digital asset readily available is usually a recreation of that asset or the additional time spent hunting down acceptable pickup art. One conservative estimate calculates the replacement value of each asset at $\$ 400$. If art must be redone for the 1,000 or so assets not found (40 per cent of $2,500)$, and each is nominally valued at $\$ 400$, the result would be a $\$ 400,000$ ROI calculation. While this is not a bad starting point to assess your own savings, the truth is that companies with successful DAM system implementations are easily achieving this and more.
One of the most important things a DAM system does is enforce a way of working with assets. It discourages ad hoc interaction with them. This can mean that assets move from an environment that encourages data silos (little mini-repositories on each designer's desktop) to a central repository where each asset is accounted for. It also means that unlike the distributed storage of assets typically found in manually administered folderbased systems, the central repository uses more efficient and consistent means to characterize assets.

An efficient, robust system today uses metadata for this purpose and it is the most important technology available for performing granular searches and tying together assets within the database that are part of composite layouts and artwork. Without the pervasive use of metadata and easy-to-use tools to attach metadata values to files, repositories cannot account for the increase in productivity you should expect to achieve. 
Access to data also refers to syndication. Once the assets are stored on a central repository, they can be accessed through a software client. In the past these clients were native UNIX, Windows or Macintosh programs. Today, the most efficient platform-neutral approach utilizes a browser-based system. The user interface remains consistent regardless of the browser used or the operating system. The process is simple, regardless of where the user is or what language is required.

Relying on a browser and using browser user interface conventions greatly reduces the changeover time and costs of moving from another system. These costs - the cost of disruption - can have an effect on the work in progress and on the overall culture of a company. While these costs can be factored into the ROI, they also fall neatly into the overall TCO and TCA.

\section{HARD COSTS AND SOFT COSTS}

ROI calculations typically focus on hard costs and soft costs. Hard costs relate to outside services that are purchased to assist in managing data. For example, shipping proofs, chromes or mockups via an overnight service are hard costs. Included in this calculation is all of the media used to store the assets such as CDs, DVDs and tapes.

Soft costs are all of the activities that go into managing and preparing for the transport of assets. Examples include burning DVDs and CDs, setting up and ripping PDF files, and assembling all of the digital assets to build the package to send out. The day-to-day activities managing the network, training users and providing support can also provide a significant soft cost to the equation.

\section{QUANTIFYING ROI}

There are two main ways of quantifying an ROI. They are:

- Payback period

- Detailed analysis of cost versus expected benefits

Payback period: This simple calculation quantifies how soon the investment in a system will be paid back. So, using one of the methods mentioned above to estimate the amount of time that will be saved by reducing searching and tangential activities related to hard and soft costs, and by breaking it down to a monthly savings before dividing it into the system cost will provide a quick but imperfect guide to the ROI of the system.

Obviously a shorter time for payback is preferred over a longer time. When placed into a broader strategic ROI context, however, a longer period may be acceptable. The ratio and range often quoted for DAM system ROI is between $8: 1$ and $14: 1$. This means for every dollar spent, the resulting savings will be $8-14$ dollars over a period of time. The period of time is the variable and takes into account the narrowly focused departmental need for the new system or the broader and more strategic corporate need for the system.

Detailed analysis of cost versus expected benefits: Strategic implications can affect the value of an investment in a DAM system. Where strategic ROI impacts the quantification of ROI is where it is part of a broader corporate initiative or product development project. Often a new system is brought on to support a new client or project and limited to assets used for that project. This provides some time for a group of eventual champions for the system to work with it in a limited way and find out how it meshes with the culture and workflow ideals of the company.

When the new system is part of a broader corporate ERP or CRM makeover, the expected benefits interlock with other departments effected in the changeover. In this case, the keystroke saving calculations of the creative group would be added to the other calculations and a case would be built for a larger system.

\section{STRATEGIC ROI}

In general, strategic ROI is expressed in the reusability of assets and the use of the core intellectual property of a company to develop new revenue streams. The reuse of assets and development of new revenue streams often happen outside the creative department where the assets may have originated. Prior to the emergence of the web as a sales and distribution tool, the notion of making money by repurposing assets was not the significant business factor it is today. 
For example, an agency with an automotive account can keep track of dealer group usage of specific assets downloaded from a DAM system through a log, then bill the client for rights and processing in an efficient way.

Examples of strategic advantages — advantages that have an impact on the corporation - are sprinkled throughout this document. They include compliance, collaboration, brand syndication, more effective use of resources in creative and IT departments, and the ability to integrate a DAM system into the general corporate data flow. What should not be overlooked in all this is that a DAM system is a unique brand of multi-media database that exists usually in an environment of other databases. At some level it is useful for these dissimilar, critical repositories to frequently communicate and synchronize data. While DAM systems serve content creation with rich media, surrounding database systems serve data that are transformed into information that can become knowledge.

New revenue opportunities can be a bit more abstract when considering a new system. It is true that hosting and managing the digital assets of a client is one way of monetizing a system. Short of doing that however is the typical selfservice functionality that web-based systems provide to enable users, wherever they are to serve themselves assets in the style and format they need for the job.

Today more and more emphasis is being placed on achieving an all-digital workflow. For example, among the hard and soft costs mentioned above were those associated with preparing and shipping proofs and media for the review and approval cycle. Time is spent, shipping costs accrued to the job and a fair amount of time lost to the lag while things are prepared and on the way. If this lag time is converted to productive review and approval time, the result is a streamlined process and increased margins. None of this can be achieved with an analog or hybrid system. They just do not scale well or promote efficiency. It can only be done with the type of file transformation automation provided by DAM systems with additional functionality for soft proofing and annotation.

The implication of a pure digital workflow that includes improved review and approval cycles is immense. It touches the client's sales (time to market), marketing collateral printing costs, web development timeframes and the betterment of customer relationships in general. This is where the advantages transcend tactical keystroke-based calculation, and relate to strategic impact. 